\title{
FACTORES DE RIESGO DE PREECLAMPSIA, UNA ACTUALIZACIÓN DESDE LA MEDICINA BASADA EN EVIDENCIAS
}

\author{
Enrique Guevara-Ríos ${ }^{1, a}$, Carlos Gonzales-Medina (1) b, c
}

\begin{abstract}
RESUMEN
La preeclampsia constituye un problema de salud pública en medicina materno perinatal y el reconocimiento de los factores de riesgo es de vital importancia para su manejo inicial. Asimismo, el vertiginoso avance de la ciencia y la investigación, nos compromete a revisar desde el enfoque actual de la medicina basada en evidencias (MBE) los nuevos hallazgos que sustentan el reconocimiento de los potenciales factores riesgo de nuestras pacientes en la práctica clínica diaria. Por ello el objetivo de la presente revisión es documentar desde el enfoque de la MBE las últimas publicaciones en relación al estudio de los factores de riesgo de preeclampsia.
\end{abstract}

Palabras clave: Preeclampsia; Factor de riesgo; Medicina basada en evidencias (Fuente: DeCS BIREME).

\section{RISK FACTORS OF PREECLAMPSIA, AN UPDATE FROM MEDICINE BASED ON EVIDENCE}

\begin{abstract}
Pre-eclampsia is a public health problem in perinatal maternal medicine and the recognition of risk factors is of vital importance for its initial management. Likewise, the vertiginous advance of science and research, commits us to review from the current approach of evidence-based medicine (EBM) the new findings that support the recognition of the potential risk factors of our patients in daily clinical practice. Therefore, the objective of this review is to document, from the MBE's perspective, the latest publications in relation to the study of risk factors for pre-eclampsia.
\end{abstract}

Keywords: Pre-eclampsia; Risk factor; Evidence-based medicine (Source: MeSH NLM).

\section{INTRODUCCIÓN}

La preeclampsia es un trastorno hipertensivo del embarazo que condiciona un mayor riesgo de morbilidad materno perinatal ${ }^{1}$. Por ello, se considera que es un problema de salud pública a nivel mundial ${ }^{2}$. Además, las investigaciones en relación a sus posibles causas todavía siguen sin ser dilucidadas por la comunidad científica. Sin embargo, el reconocimiento de las probables etiologías se deja de lado por desarrollar investigaciones sobre el manejo y el diagnostico precoz ${ }^{3}$. La razón es que desde el punto de vista práctico estas dos condiciones (el manejo y el diagnostico precoz) generan mayores complicaciones. Así pues, desde un enfoque epidemiológico la identificación de los factores de riesgo potenciales se puede orientar de forma integral y multidisciplinaria ${ }^{4}$. Según la Organización Mundial de la Salud (OMS) un factor de riesgo es todo rasgo, característica o exposición de un individuo que aumenta su probabilidad de sufrir una enfermedad o lesión ${ }^{5}$. Esta característica propia de cada individuo puede convertirse en una condición que más adelante le predisponga a desarrollar una enfermedad $y$, por lo tanto, esta característica puede convertirse en un factor de riesgo. Situación no ajena a la que ocurre en la preeclampsia ${ }^{5}$. Por ello, en las gestantes con preeclampsia, muchas veces el riesgo se identifica, por ejemplo, desde el reconocimiento de un embarazo previo con preeclampsia ${ }^{6}$. Esta condición, supone que el especialista, evaluador del control prenatal, considere los potenciales riesgos de la paciente y oriente su enfoque basado en la disminución e identificación de potenciales desencadenantes de la enfermedad ${ }^{7}$. Resulta interesante que el profesional de la salud a cargo del control prenatal conozca, a la luz de los nuevos hallazgos de la medicina basada en la evidencia (MBE) las probabilidades del riesgo de desarrollar preeclampsia en el embarazo actual y oriente a la paciente sobre este problema. Dato muy importante que la corriente del MBE denomina, la toma de decisiones ${ }^{8}$. Por ello, el objetivo primario de esta revisión es documentar desde el enfoque de la MBE las últimas

\footnotetext{
Médico Gíneco-Obstetra. Director del Instituto Nacional Materno Perinatal. Lima-Perú. Coordinador de Asistencia Técnica del Instituto de Salud Popular, Consultor de Pathfinder International. Lima-Perú.

a Profesor de Facultad de Medicina de la Universidad Nacional Mayor de San Marcos Lima-Perú.

2 Médico Cirujano Especialista en Ginecología y Obstetricia Facultad de Medicina San Fernando, Universidad Nacional Mayor de San Marcos (UNMSM), Miembro de la Sociedad Peruana de Ginecología y Obstetricia SPOG, Perú. Fellow Junior ACOG, American College of Obstetricians and Gynecologists, United States of America.

Código ORCID: Carlos Gonzales Medina @ https://orcid.org/0000-0002-9840-1886

b Unidad Funcional de Investigación del Instituto Nacional Materno Perinatal- Lima, Perú.

Maestría en Investigación Clínica, Universidad San Martin de Porres. Lima-Perú.
}

Citar como: Guevara E, Gonzales-Medina C. Factores de riesgo de preeclampsia, una actualización desde la medicina basada en evidencias. Rev Peru Investig Matern Perinat 2019; 8(1):30-5.

DOI: https://doi.org/10.33421/inmp.2019140

Recibido: 07-03-19 Aprobado: 27-03-19 
publicaciones y aquellas que mantienen una jerarquía como estudios primarios en relación al estudio de los factores de riesgo de preeclampsia.

\section{DEFINICIÓN DE PREECLAMPSIA}

La preeclampsia se define como parte de un grupo de entidades denominadas los desórdenes hipertensivos del embarazo ${ }^{9}$. Una clasificación que no ha variado desde la publicación de la Working Group del $2008^{10}$. Y su definición conceptual actual es la siguiente: La preeclampsia es la aparición de un nuevo inicio de hipertensión arterial con proteinuria o hipertensión arterial acompañado de un daño multiorgánico con o sin proteinuria que aparece posterior a las 20 semanas de embarazo o en el post parto en una gestante que previamente era normotensa o tenía alguna condición de riesgo que la predisponga a desarrollar la enfermedad ${ }^{11}$.

\section{CRITERIOS DIAGNÓSTICOS Y CRITERIOS DE PREECLAMPSIA SEVERA}

Desde la publicación de ACOG en el año 2013, se consideró excluir a la proteinuria significativa mayor de $300 \mathrm{mg}$ en orina de 24 horas como criterio independiente de preeclampsia severa. Y es que a la luz de las evidencias y en la práctica diaria los criterios de la característica severa anteriores se comportaban independientemente de la aparición del daño renal establecido ${ }^{11}$. En la tabla 1 y la tabla 2 se detallan los criterios de severidad y diagnósticos documentados por el ACOG en su última revisión del $2019^{12}$.

Tabla 1. Criterios diagnósticos de preeclampsia ${ }^{12}$

\begin{abstract}
Elevación de la presión arterial sistólica con cifras $\geq 140$ $\mathrm{mmHg}$ o elevación del presión arterial diastólica con cifras mayores a $\geq 90 \mathrm{mmHg}$ tomadas en dos ocasiones con un intervalo de 4 horas de separación en un embarazo mayor de 20 semanas en la que previamente la paciente era normotensa y con la presencia inicial de una o más de las siguientes características $\left({ }^{*}\right)$ :

- $\quad$ Proteinuria $\geq 0,3 \mathrm{~g}$ en orina de 24 horas o un índice proteína/creatinina $\geq 0,3(\mathrm{mg} / \mathrm{mg})(30 \mathrm{mg} / \mathrm{mmol})$ en una muestra de orina al azar o una medición de tira reactiva $\geq$ +2 si una medida cuantitativa no está disponible.

- Conteo de plaquetas < 100 000/ microL

- Creatinina en suero > $1.1 \mathrm{mg} / \mathrm{dL}(97.2 \mathrm{micromol} / \mathrm{L})$ o el doble del valor de creatinina sérica en ausencia de alguna enfermedad renal

- Elevación al doble de su límite normal de las enzimas hepáticas medias en laboratorio

\section{- Edema pulmonar}

- Síntomas cerebrales o disturbios visuales (Por ejemplo, nuevo inicio de cefalea persistente no identificada por diagnósticos previos o diferenciales y que no responde a la dosis habitual de analgésicos, visión borrosa, escotomas, luces o chispas parpadeantes
\end{abstract}

${ }^{*}$ ) Si la presión arterial sistólica es mayor de $\geq 160 \mathrm{mmHg}$ o la presión arterial diastólica es mayor de $\geq 110 \mathrm{mmHg}$ confirmados en minutos con otra toma el criterio es suficiente.
Tabla 2. Criterios de preeclampsia severa, la presencia de una o más de las siguientes condiciones indican el diagnostico de preeclampsia con características de severa $\left({ }^{*}\right)$

\begin{tabular}{l}
\hline a. Elevación severa de la presiona arterial (£) \\
La presión arterial sistólica es mayor de $\geq 160 \mathrm{mmHg}$ o la \\
presión arterial diastólica es mayor de $\geq 110 \mathrm{mmHg}$ medidos en \\
dos ocasiones con un intervalo de 4 horas mientras el paciente \\
se hallaba en cama (la terapia antihipertensiva puede iniciarse \\
ante la aparición de hipertensión grave y puede establecerse \\
como criterio de severidad sin esperar las cuatro horas de \\
diferencia) \\
b. Síntomas de disfunción del sistema nervioso central \\
Nuevo inicio (inicio brusco) de disturbios visuales tales como: \\
- Fotopsias, escotomas, ceguera cortical y vasoespasmo \\
retinal \\
Cefalea severa (Es decir incapacitante "El peor dolor de \\
cabeza que he tenido") o cefalea persistente y progresiva a \\
pesar de la terapia analgésica y no hallar otros diagnósticos \\
diferenciales.
\end{tabular}

\section{c. Anormalidades Hepáticas}

Dolor persistente severo en cuadrante superior derecho o epigástrico que no responde a la medicación en los que no se halla otros diagnósticos diferenciales o la elevación de la concentración hepática de transaminasas mayor igual a dos veces su límite superior normal o ambos.

\section{d. Trombocitopenia}

Numero de plaquetas Menor a $<100000$ platelets/microL

\section{e. Anormalidades renales}

Insuficiencia renal (valores de creatinina > $1.1 \mathrm{mg} / \mathrm{dL}[97.2$ micromol/L] o el doble del valor de creatinina sérica en ausencia de alguna enfermedad renal)

\section{f. Edema Pulmonar}

(£) Para el caso de una paciente que tiene una hipertensión relacionada con el embarazo los criterios de característica de preeclampsia severa se cumplen cuando se cumplen los criterios de los ítems $\boldsymbol{b}, \boldsymbol{c}, \boldsymbol{d}$, e (se excluye edema pulmonar y en el caso de la presión arterial se considera presión arterial sistólica $\geq 160 \mathrm{mmHg}$ o la presión arterial diastólica $\geq 110 \mathrm{mmHg}$ o ambos medidos en dos ocasiones, no se considera el tiempo u intervalo.

\section{FACTORES DE RIESGO}

Según las últimas revisiones del Colegio Americano de Ginecología y Obstetricia (ACOG) y las establecidas en las directrices actualizadas en UptoDate hasta junio del 2019 los factores de riesgo de preeclampsia se resumen en la figura 1. Asimismo, se muestra los factores clínicos que se asocian con un elevado riesgo de desarrollo de preeclampsia $^{12}$.

Es importante recordar que actualmente la descripción de los factores riesgo de preeclampsia se aplican tanto para la preeclampsia de inicio temprano como para la preeclampsia de inicio tardío. Aunque existen algunas condiciones propias de las pacientes o enfermedades que condicionan a la aparición de una u otra característica como es el caso del Lupus Eritematosos Sistémico activo que está más asociado con la aparición o recurrencia de los casos de preeclampsia de inicio temprano y es considerado en los nomogramas de predicción actuales para su predicción ${ }^{13}$. 
Factores clínicos que se han asociado con un mayo riesgo de desarrollar preeclampsia

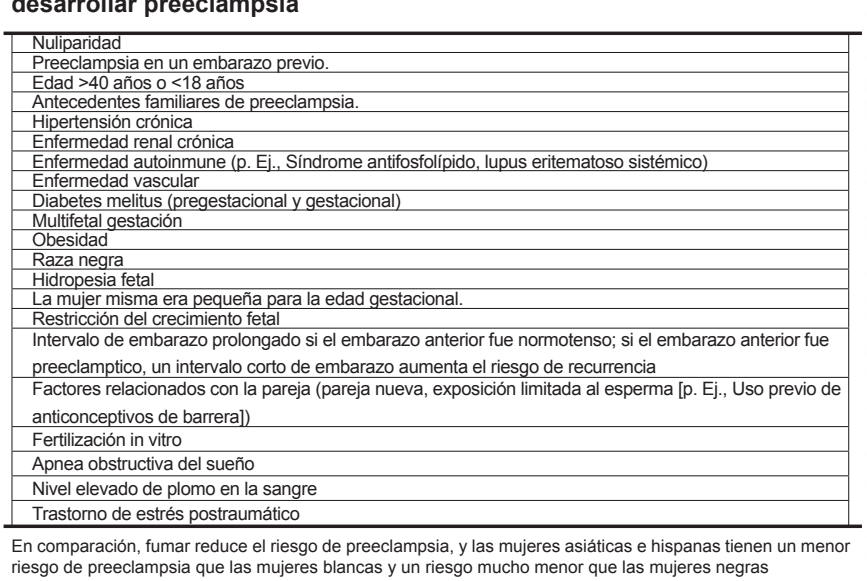

riesgo de preeclampsia que las mujeres blancas y un riesgo mucho menor que las mujeres negras

A

\section{Factores de riesgo de preeclampsia}

\section{- Nuliparidad}

- Gestación múltiple

- Preeclampsia en gestaciones previas

- Hipertensión crónica

- Diabetes gestacional

- Trombofilias hereditarias

- Lupus eritematoso sistémico

- Índice de masa corporal pregestacional mayor de 30

- Síndrome antifosfolípido

- Edad materna mayor de 35 años

- Enfermedad de renal

- Terapia de reproducción asistida

- Apnea obstructiva del sueño

Figura 1. (A) Factores clínicos que se asocian con un mayor riesgo de desarrollo de preeclampsia según las últimas actualizaciones de Uptodate. (B) Factores de riesgo de preeclampsia según el Colegio Americano de Ginecología y Obstetricia (ACOG)

Por ello durante la descripción de cada uno de los factores de riesgo clínico se mostraran las evidencias de la asociación, la explicación desde sus bases teóricas y la fisiopatología. Y finalmente, cuál es la referencia que generó la mejor evidencia en relación a este factor asociado que potencialmente se considera como factor de riesgo de la enfermedad.

A continuación se desarrollaran los factores asociados desde una perspectiva de la Medicina Basada en la Evidencias mostrando en el análisis de la lectura crítica la mejor evidencia disponible según la actualización del centro para la MBE de Oxford.

\section{Nuliparidad}

La asociación entre la nuliparidad y la preeclampsia: Un aspecto interesante de esta asociación es que desde el inicio de la documentación de la enfermedad esta era conocida como la "enfermedad del primer embarazo". Y es que en aquellas mujeres que ya tenían un embarazo previo sin complicaciones, la frecuencia de aparición de la enfermedad era muy baja. Asimismo, la explicación fisiopatológica se debería a una mala adaptación inmunológica a nivel placentario en la mujer nulípara como hipótesis básica para explicar la ocurrencia de preeclampsia. Y la mejor evidencia disponible menciona que el riesgo relativo $(R R)$ en mujeres nulíparas es RR: 2.1 (95\% Cl, 1.9-2.4) en estudios observacionales de cohortes ${ }^{14}$.

\section{Preeclampsia en embarazos previos}

Merece especial atención la historia personal de preeclampsia, la historia familiar de preeclampsia y la historia del padre con una mujer que previamente embarazó y que desarrollo preeclamsia. La historia de preeclampsia como factor previo es interesante. La asociación con el antecedente previo de historia de preeclampsia considerado tal vez uno de los escenarios en los que la probabilidad del riesgo se incrementa estadísticamente más y por ello en la toda la literatura médica se cataloga como "el predictor más fuerte" de desarrollo de la enfermedad. Así, se determina que el riesgo de desarrollar preeclampsia en el embarazo actual de una paciente que previamente tuvo preeclampsia es de aproximadamente $20 \%{ }^{2}$. Y este riesgo puede oscilar entre un 5 y $80 \%$ dependiendo del momento de aparición y de la severidad documentada previamente ${ }^{15}$. Asimismo, la explicación fisiopatológica es claramente atribuida a la carga genética según diversos estudios. Sin embargo, una característica génica heredada de tipo somática también se asocia particularmente con el desarrollo de la enfermedad. Por ejemplo, en un estudio japonés se identificó que la talla menor de $155 \mathrm{~cm}$ se asociaba fuertemente al desarrollo de preeclampsia en una cohorte de seguimiento familiar con un (RR, 1.35; 95\% Cl, 1.25-1.45) $)^{15}$. Por tanto el gen somático que determina la talla estaría involucrado en el desarrollo de forma hereditario de preeclampsia Además, la mejor evidencia sostenible menciona que el riesgo relativo (RR) ajustado para la historia previa de preeclampsia es de $8.4(95 \% \mathrm{Cl}, 7.1-9.9)$ en grandes revisiones sistemáticas sobre esta condición ${ }^{2}$.

En cuanto a si la historia familiar de la paciente en la gestación actual cobra importancia a la luz de las evidencias de estudios en familias. $Y$ es que aquellas madres, hermanas, tías y abuelas en las que se suscitó el cuadro la probabilidad de aparición del evento es cercano al triple en comparación con aquellas que no tienen este antecedente. Sin embargo resulta más interesante entender que según la aparición de la preeclampsia en la gestación previa el riesgo aumenta mucho más si la aparición previa fue más precoz. Así el riesgo de desarrollar preeclampsia en la gestación actual si en la gestación anterior de la historia familiar inicio temprano, intermedio y tardío se incrementa progresivamente

\section{Edad mayor de 40 años o menor de 18 años}

Edad materna avanzada es considerado epidemiológicamente un factor de riesgo importante (edad materna $\geq 35$ años, RR 1.2, IC 95\% 1.1-1.3 y $\geq 40$ años, RR 1.5, IC 95\% 1.2-2.0) ${ }^{16}$. Las mujeres mayores tienden a tener factores de riesgo adicionales, como la diabetes mellitus, la hipertensión crónica, o alguna 
enfermedad crónica que las predispone a desarrollar preeclampsia. En el caso de las adolescentes el riesgo es mayor estadísticamente pero en muchos estudios observacionales el riesgo de preeclampsia no es claro del todo ${ }^{17}$ una revisión sistemática no encontró una asociación de forma directa y en las revisiones actuales de ACOG no se considera como un factor de riesgo en sus reportes ${ }^{15}$.

\section{Familia con historia de preeclampsia}

Una historia familiar de preeclampsia en un pariente de primer grado eleva el riesgo de preeclampsia ( $R R$ $2,90$, IC del $95 \% 1,70$ a 4,93$)^{15}$ lo que sugiere un mecanismo heredable en algunos casos sobre todo a partir de estudios de polimorfimos de genes claramente documentados como del Factor $\mathrm{V}$ de Leiden, el de los antígenos leucocitarios HLA, de las alteraciones NOS 3 del óxido nítrico endotelial, el angiotensinógeno y de la lipoproteinlipasa ${ }^{18}$. La aparición y la gravedad de la enfermedad parecen estar influenciadas principalmente por factores maternos, pero la contribución paterna a los genes fetales puede tener un papel en la placentación defectuosa y la preeclampsia posterior ${ }^{19}$.

\section{Hipertensión crónica}

También se ha informado que la hipertensión crónica tiene un papel preponderante en la predisposición de las pacientes al desarrollo de preeclampsia (RR 5.1, IC $95 \%$ 4.0-6.5 $)^{16}$. Hay información en relación a los valore de presión arterial $\geq 130 / 80 \mathrm{mmHg}$ en la primera visita prenatal que aumentan el riesgo que en estudios de seguimiento han demostrado una asociación importante (RR 1.38 a 2.37) ${ }^{15}$. Aunque la hipertensión crónica (definida como presión arterial $\geq 140 / 90 \mathrm{mmHg}$ ) aumenta cinco veces el riesgo de preeclampsia en comparación con las mujeres sin este factor de riesgo, la hipertensión crónica es poco común en mujeres en edad reproductiva y, por lo tanto, representa solo del 5 al 10 por ciento de los casos de preeclampsia ${ }^{20}$. Asimismo. el Colegio Americano de Cardiología y la Asociación Americana del Corazón revisó sus definiciones de hipertensión en el 2017 y los datos preliminares sugieren que las mujeres con presiones sistólicas de 130 a 135 y/o las presiones diastólicas de 80 a $85 \mathrm{mmHg}$ también tienen un mayor riesgo importante de preeclampsia ${ }^{21}$.

\section{Enfermedad renal crónica}

Enfermedad renal crónica (ERC) tiene un riesgo demostrado a partir de los estudios epidemiológicos y también por la alteración fisiopatológica del daño renal alterado por la hiperfiltracion de proteínas y la consecuente caída de la presión oncótica en los vasos capilares. El riesgo basal de toda paciente con una ERC es cercano a dos en los estudios observacionales de cohortes (RR 1.8, IC 95\% 1.5-2.1 $)^{16}$. Asimismo, el riesgo varía según el grado de reducción de la tasa de filtración glomerular y la presencia o ausencia de hipertensión. En algunos estudios, hasta 40 a 60 por ciento de las mujeres con ERC avanzada (estadios 3, 4 y 5) fueron diagnosticadas con preeclampsia en la segunda mitad del embarazo ${ }^{22}$.
Enfermedad autoinmune (Síndrome Antifosfolípidico y Lupus Eritematoso sistémico)

Cuando se evaluó los casos de síndrome antifosfolípido se determinó que existe un riesgo relativo cercano a tres en relación a las pacientes que no sufren esta condición para el desarrollo de preeclampsia. Así se estima un RR 2.8, IC $95 \% 1.8-4.3^{16}$. El lupus eritematoso sistémico una condición que implica una alteración en la función de los linfocitos T Hellper directamente se agrava por las modificaciones del embarazo y predispone a una disfunción inmunológica que agrava y desencadena la preeclampsia. Se estima que las pacientes que sufren esta condición tienen un RR 1,8, IC 95\% 1.5 a 2.1 cuando se analizan los estudios observacionales de cohortes prospectivas.

\section{Diabetes Mellitus y Diabetes pregestacional}

Existen múltiples datos que documentan desde estudios poblacionales previos que la diabetes gestacional se asocia claramente con el desarrollo de preeclampsia (OR ajustado 1,61; IC 95\%: 1,39 a 1,89 $)^{23}$. En recientes estudios longitudinales se data que el RR 3.7, IC 95\% 3.1-4.3 (16). Un ejemplo, es el reportado en un seguimiento de 82436 gestantes en el que se halló que la diabetes era un factor de riesgo importante para el desarrollo de preeclampsia (OR 5,64; IC95\%: 4,33 a 7,35) ${ }^{24}$. La explicación fisiopatológica es claramente atribuida al aumento de una variedad de factores implicados, como la enfermedad renal o vascular subyacente, los niveles altos de insulina en plasma así como la resistencia a la insulina y el metabolismo anormal de los lípidos ${ }^{25}$. Un dato interesante para considerar es que en el Perú en una población de limeña de gestantes el antecedente de diabetes mellitus en por lo menos uno de los padres tuvo un riesgo de 3,4 veces (IC 95\%: 1,4 a 8,4 ) mayor probabilidad de presentar preeclampsia y que el antecedente de tener familiares con diabetes o con hipertensión arterial generó un aumento en el indicador de riesgo con un OR de 4,6 (IC 95\%: 0,9 a 23,0). Sin embargo, esta diferencia, como señalan los autores, no fue significativa dada la pequeña cantidad de $\operatorname{casos}^{26}$.

\section{Embarazo multifetal}

Embarazo multifetal como condición obstétrica de alto riesgo por la alteración placentaria y los mecanismos inmunológicos propios de la disfunción placentaria y endotelial genera un riesgo cercano a tres veces en comparación con los embarazos únicos (RR 2.9, IC 95\% 2.6-3.1) ${ }^{27}$.El riesgo aumenta al aumentar el número de fetos, así lo demuestra un estudio de casos y controles en relación al cálculo del riesgo de preeclampsia. En el cual se determinó que es mayor en el embarazo tripe en comparación con el doble con un OR: 4,9 IC95\%(1.2-23.5 $)^{28}$.

\section{Obesidad}

El índice de masa corporal antes del embarazo con cifras mayores a $25 \mathrm{~kg} / \mathrm{m} 2$ genera un aumento del riesgo de desarrollar preeclampsia (RR 2.1, IC 95\% 2.0-2.2). Asimismo, el índice de masa corporal (IMC) mayor de $30 \mathrm{~kg} / \mathrm{m} 2$ (RR 2.8, IC 95\% 2.6-3.1 $)^{29}$. El riesgo de preeclampsia se duplica con cada 5 a $7 \mathrm{~kg} / \mathrm{m} 2$ de aumento del IMC antes del embarazo ${ }^{2}$. 
Cuando se desarrolló un estudio de cohortes prospectiva en base comunitaria en el que se realizó un seguimiento a 3840 mujeres con obesidad mórbida se determinó que el riesgo es cercano a 5 veces en relación a los controles normotensos (OR 4,82; IC95\%: 4,04 a 5,74)². Esta relación persistió en estudios que excluyeron a las mujeres con hipertensión crónica, diabetes mellitus, gestaciones múltiples o después del ajuste para otros factores de confusión. Aunque el sobrepeso y la obesidad aumentan el riesgo de preeclampsia solo de dos a tres veces, el sobrepeso y la obesidad son altamente prevalentes en todo el mundo y, por lo tanto, representan más del 40 por ciento de los casos de preeclampsia ${ }^{20}$. La explicación fisiopatológica es claramente derivada de la disfunción del metabolismo de los lípidos y del síndrome metabólico asociado con los factores antiangiogenicos expresados productos de la disfunción endotelial como son el sflt-1 y PIGF 30 .

Factores relacionados con la paternidad (Nueva pareja, exposición limitada al esperma como por ejemplo el uso de métodos de barreras para evitar la contracepción)

Una teoría es que el sistema inmunológico de las mujeres nulíparas ha tenido una exposición limitada a los antígenos paternos, y esta falta de desensibilización puede jugar un papel en la patogenia de la enfermedad en general este factor tiene un riesgo de dos (RR 2.1, IC 95\% 1.9-2.4) 16 $^{\text {. Los }}$ datos epidemiológicos apoyan esta teoría: la protección contra la preeclampsia en embarazos subsiguientes se reduce o se elimina si hay un cambio en la paternidad, las mujeres que usan métodos anticonceptivos de barrera tienen un riesgo mayor y el riesgo se reduce al aumentar la duración de la actividad sexual antes del embarazo ${ }^{14}$. Sin embargo, la noción de que el riesgo de preeclampsia aumenta en un segundo embarazo con una nueva pareja ha sido cuestionada por datos que sugieren que un intervalo más prolongado entre embarazos puede ser la razón del mayor riesgo con una nueva pareja ${ }^{14}$.

\section{Fertilización in vitro}

Uso de tecnología de reproducción asistida es un factor de riesgo en los estudios de cohortes de gran tamaño (tasa combinada del 6.2 por ciento, IC 95\% 4.7-7.9; RR 1.8, IC 95\% 1.6-2.1 $)^{16}$. Sin embargo, el análisis de regresión logística multivariante atenúa esta asociación y el análisis de la propensión lo debilita aún más pero se ha visto una fuerte asociación entre esta tecnología y el desarrollo de preeclampsia.

\section{Apnea obstructiva del sueño}

Si bien la literatura actual documenta a la apnea obstructiva del sueño como una condición de riesgo de preeclampsia hasta hace una década esta condición aún no se consideraba como un factor de riesgo basado en la evidencia. Es a raíz de las hipótesis fisiológicas de la fisiopatología del sueño alterado como resultado de la limitación al flujo aéreo, la corta duración del sueño y las desaturaciones que se suscitaría una predisposición materna a un cuadro hipoxico que activaría por mecanismos inmunológicos y metabólicos a los factores de expresión del daño endotelial y sistémico. Las respuestas clásicamente estudiadas durante el embarazo entonces son la disfunción endotelial, la hiperactividad sistémica, la inflamación sistémica y la desregulación metabólica ${ }^{31}$.La evidencia documenta que las pacientes que cursan con apnea obstructiva del sueño tiene cerca de dos veces el riesgo de desarrollar preeclampsia en comparación con los controles (OR 1.6, IC 95\% 1.28-2.40) ). $^{32}$.

Complicaciones previas del embarazo asociadas con insuficiencia placentaria: La restricción del crecimiento fetal tiene un riesgo de desarrollar preeclampsia en mucha series y estudios observacionales (RR 1.4, IC 95\% 0.6-3.0). Asimismo, la muerte fetal como evento adverso durante el embarazo se asocia y es considerado un factor de riesgo de preeclampsia RR 2.4, IC 95\% 1.7-3.4 ${ }^{16}$. Todos los mecanismos inmunológicos debidos a la insuficiencia placentaria y su la subsiguiente disfunción endotelial llevan a un proceso de hipoxia crónica fetal la desviación de la curva de disociación de la hemoglobina fetal y la alteración en la distribución del oxígeno para los proceso anabólicos del crecimiento fetal. Así mismo, es interesante entender que el desbalance del cociente sFIt-1/PIGF recientemente se halla como un probable predictor y diagnóstico de la enfermedad sobre en el embarazo cercano al término hasta incluso 5 semanas antes que se diagnostique la preeclampsia. $Y$ por lo tanto, este cociente promete ser un interesante marcador del manejo y pronóstico de la preeclampsia ${ }^{33}$

Debemos mencionar que los factores de riesgo establecidos por la literatura médica están diseñados de grandes estudios observacionales de cohortes y casos y controles. Asimismo, hay grandes estudios transversales que datan evidencia de alta calidad. Uno de los factores estudiados y que tienen buena evidencia recientemente también es la violencia basada en el género ${ }^{34}$, el hipotiroidismo subclínico ${ }^{35}$ y la obesidad durante el embarazo no evaluada ${ }^{36}$.

En conclusión, los factores de riesgo clínicos descritos por la ACOG y los desarrollados por Uptodate determinan la capacidad analítica de los autores y actualiza la información reciente según lo conocido en la literatura. Y lo más interesante es que se establece a partir del conocimiento de la mejor evidencia disponible. Claramente son los factores de riesgo la nuliparidad, la gestación múltiple, la preeclampsia en gestaciones previas, la hipertensión crónica, la diabetes gestacional, las trombofilias hereditarias, el lupus eritematoso, el índice de masa corporal pregestacional mayor de 30, la presencia de síndrome antifosfolípido, la edad materna avanzada mayor de 35 años, la enfermedad de renal, los embarazo en los que se usó la tecnología de reproducción asistida y el apnea obstructiva del sueño.

Financiamiento: Autofinanciado.

Conflicto de interés: Los autores declaran no tener algún conflicto de intereses. 


\section{REFERENCIAS BIBLIOGRÁFICAS}

1. Duley L. The global impact of pre-eclampsia and eclampsia. Semin Perinatol. junio de 2009;33(3):130-7.

2. Conde-Agudelo A, Belizán JM. Risk factors for pre-eclampsia in a large cohort of Latin American and Caribbean women. BJOG Int J Obstet Gynaecol. 2000;107(1):75-83.

3. Emonts $P$, Seaksan S, Seidel L, Thoumsin H, Gaspard $\mathrm{U}$, Albert A, et al. Prediction of maternal predisposition to preeclampsia. Hypertens Pregnancy. 2008;27(3):237-45.

4. Gonzales Medina CA, Alegría Guerrero CR. ¿Es posible predecir la preeclampsia? Rev Peru Ginecol Obstet. octubre de 2014;60(4):363-72.

5. Pacheco J, Wagner P, Williams M, Sánchez S. Enfermedades hipertensivas en la gestación. En: Pacheco J Ginecología, Obstetricia y Reproducción. 2da ed. Lima: REP-SAC; 2007. p. 1097-130.

6. Lisonkova S, Joseph KS. Incidence of preeclampsia: risk factors and outcomes associated with early- versus late-onset disease. Am J Obstet Gynecol. 2013;209(6):544.e1-544.e12.

7. Sánchez SE. Actualización en la epidemiología de la preeclampsia: update. Rev Peru Ginecol Obstet. octubre de 2014;60(4):309-20.

8. Segundo-Paredes JA, Gonzales-Medina CA, Francia-Dela-Cruz RJ, Valdivia-Vera E, Mejía-Veramendi JP, ArangoOchante PM. Conocimientos, actitudes y prácticas de la medicina basada en evidencias en médicos asistentes de un hospital público. Lima- Perú. Rev Peru Investig Materno Perinat. 2018;7(2):9-15.

9. Cudihy D, Lee RV. The pathophysiology of pre-eclampsia: current clinical concepts. J Obstet Gynaecol J Inst Obstet Gynaecol. octubre de 2009;29(7):576-82.

10. Williams D. Long-term complications of preeclampsia. Semin Nephrol. enero de 2011;31(1):111-22.

11. American College of Obstetricians and Gynecologists, Task Force on Hypertension in Pregnancy. Hypertension in pregnancy. Report of the American College of Obstetricians and Gynecologists' Task Force on Hypertension in Pregnancy. Obstet Gynecol. noviembre de 2013;122(5):1122-31.

12. ACOG Practice Bulletin No. 202: Gestational Hypertension and Preeclampsia. Obstet Gynecol. enero de 2019;133(1):e1-25.

13. Alegría-Guerrero R, Gonzales-Medina C. Evaluación diagnóstica de un nomograma de predicción de preeclampsia. Rev Peru Investig Materno Perinat. 2018;7(2):21-30.

14. Skjærven R, Wilcox AJ, Lie RT. The Interval between Pregnancies and the Risk of Preeclampsia. N Engl J Med. 2002;346(1):33-8.

15. Duckitt K, Harrington D. Risk factors for pre-eclampsia at antenatal booking: systematic review of controlled studies BMJ. 2005;330(7491):565

16. Bartsch E, Medcalf KE, Park AL, Ray JG. Clinical risk factors for pre-eclampsia determined in early pregnancy: systematic review and meta-analysis of large cohort studies. BMJ. 2016;11753

17. Saftlas AF, Olson DR, Franks AL, Atrash HK, Pokras R. Epidemiology of preeclampsia and eclampsia in the United States, 1979-1986. Am J Obstet Gynecol.1990;163(2):460-5.

18. Tomimatsu T, Mimura K, Endo M, Kumasawa K, Kimura T. Pathophysiology of preeclampsia: an angiogenic imbalance and long-lasting systemic vascular dysfunction. Hypertens Res Off J Jpn Soc Hypertens. 2017;40(4):305-10.

19. Lain KY, Roberts JM. Contemporary concepts of the pathogenesis and management of preeclampsia. JAMA. 26 de junio de 2002;287(24):3183-6.

20. Roberts JM, Redman CWG. Global Pregnancy Collaboration symposium: Prepregnancy and very early pregnancy antecedents of adverse pregnancy outcomes: Overview and recommendations. Placenta. diciembre de 2017;60:103-9.

21. Sutton EF, Hauspurg A, Caritis SN, Powers RW, Catov JM. Maternal Outcomes Associated With Lower Range Stage 1 Hypertension. Obstet Gynecol. 2018;132(4):843-9.

22. Blom K, Odutayo A, Bramham K, Hladunewich MA. Pregnancy and Glomerular Disease: A Systematic Review of the Literature with Management Guidelines. Clin J Am Soc Nephrol CJASN. 2017;12(11):1862-72.

23. Mack LR, Tomich PG. Gestational Diabetes: Diagnosis, Classification, and Clinical Care. Obstet Gynecol Clin North Am. junio de 2017;44(2):207-17.

24. Funai EF, Paltiel OB, Malaspina D, Friedlander $Y$, Deutsch L, Harlap S. Risk factors for pre-eclampsia in nulliparous and parous women: the Jerusalem perinatal study. Paediatr Perinat Epidemiol. enero de 2005;19(1):59-68.

25. Lee J, Ouh Y-T, Ahn KH, Hong SC, Oh M-J, Kim H-J, et al. Preeclampsia: A risk factor for gestational diabetes mellitus in subsequent pregnancy. PloS One. 2017;12(5):e0178150.

26. Sanchez SE, Zhang C, Qiu C-F, Williams MA. Family history of hypertension and diabetes in relation to preeclampsia risk in Peruvian women. Gynecol Obstet Invest. 2003;56(3):128-32.

27. Bergeron TS, Roberge S, Carpentier C, Sibai B, McCawBinns A, Bujold E. Prevention of Preeclampsia with Aspirin in Multiple Gestations: A Systematic Review and Meta-analysis. Am J Perinatol. 2016;33(6):605-10.

28. Mastrobattista JM, Skupski DW, Monga M, Blanco JD, August $P$. The rate of severe preeclampsia is increased in triplet as compared to twin gestations. Am J Perinatol. 1997;14(5):263-5.

29. van Rijn BB, Hoeks LB, Bots ML, Franx A, Bruinse HW. Outcomes of subsequent pregnancy after first pregnancy with early-onset preeclampsia. Am J Obstet Gynecol. septiembre de 2006;195(3):723-8.

30. Herraiz I, Llurba E, Verlohren S, Galindo A, Spanish Group for the Study of Angiogenic Markers in Preeclampsia. Update on the Diagnosis and Prognosis of Preeclampsia with the Aid of the sFlt-1/ PIGF Ratio in Singleton Pregnancies. Fetal Diagn Ther. 2018;43(2):81-9.

31. Shamsuzzaman ASM, Gersh BJ, Somers VK. Obstructive sleep apnea: implications for cardiac and vascular disease. JAMA. 2003;290(14):1906-14.

32. Chen Y-H, Kang J-H, Lin C-C, Wang I-T, Keller JJ, Lin H-C. Obstructive sleep apnea and the risk of adverse pregnancy outcomes. Am J Obstet Gynecol. 2012;206(2):136.e1-136.e5.

33. Ding G, Liping L, Moli D, Wuliyeti A, Shaohe Z, Huijuan W, et al. A study of the association between the sFIt-1/PIGF ratio and preeclampsia in Xinjiang Uygur Autonomous Region of China. Artif Cells Nanomedicine Biotechnol. 2018;46(sup3):S281-6.

34. Sánchez SE. Actualización en la epidemiología de la preeclampsia: update. Rev Peru Ginecol Obstet. 2014;60(4):309-20.

35. North RA, Taylor RN. Subclinical hypothyroidism after preeclampsia. BMJ. 8 de diciembre de 2009;339:b5183.

36. Fernández Alba JJ, Mesa Páez C, Vilar Sánchez Á, Soto Pazos E, González Macías MDC, Serrano Negro E, et al. [Overweight and obesity at risk factors for hypertensive states of pregnancy: a retrospective cohort study]. Nutr Hosp. 2018;35(4):874-80.

\section{Correspondencia:}

Dirección: Alameda Eugenio Yacolevf Edificio 120, Dpto: 204 La Torres de San Borja

Correo electrónico: cgonzales@iemp.gob.pe

Teléfono: 996856039 